\title{
Adaptive Swarm Robot Region Coverage Using Gene Regulatory Networks
}

\author{
Hyondong Oh and Yaochu Jin \\ Department of Computing, University of Surrey \\ Guildford, Surrey, GU2 7XH, UK \\ $\{\mathrm{h} . \mathrm{oh}$, yaochu.jin\}@surrey.ac.uk
}

\begin{abstract}
This paper proposes a morphogenetic pattern formation approach for collective systems to cover a desired region for target entrapment. This has been achieved by combining a two-layer hierarchical gene regulatory network (H-GRN) with a region-based shape control strategy. The upper layer of the H-GRN is for pattern generation that provides a desired region for entrapping targets generated from local sensory inputs of detected targets. This pattern is represented by a set of arc segments, which allow us to form entrapping shape constraints with the minimum information that can be easily used by the lower layer of the H-GRN. The lower layer is for region-based shape control consisting of two steps: guiding all robots into the desired region designated by the upper layer, and maintaining a specified minimum distance between each robot and its neighbouring robots. Numerical simulations have been performed for scenarios containing either static and moving targets to validate the feasibility and benefits of the proposed approach.
\end{abstract}

Keywords: Pattern formation, Target entrapment, Swarm robots, Gene regulatory networks

\section{Introduction}

Developing self-organising multi-agent systems has become a primary research area in recent decades due to their attractive properties such as robustness to faults and damages, adaptability to unknown environments and cost efficiency. In particular, considerable attention has been paid to multi-robot shape or region formation as a basic functionality for achieving the missions such as search and rescue, deployment of sensor network, and collective transport. Pattern formation algorithms for swarm robots can be largely divided into four categories: 1) behaviour-based control, where behavioural rules are employed onto individual robots with relative importance $[1,2] ; 2)$ leader-follower and virtual structures where the (virtual) leaders are identified and the followers follow the leaders with a set of formation constraints [3,4]; 3) potential field in which the robot moves through the gradient of a potential field defined by a sum of attractive and repulsive forces [5,6]; and 4) biologically-inspired approaches such as using morphogen gradient $[7,8]$, a pheromone/hormone model [9] or a gene regulatory network (GRN) [10,11]. 
Despite the large body of research on multi-robot pattern formation, relatively little work has been performed on the control of a large number of robots for generating adaptive shapes interacting with an unknown and dynamic environment. Swarm Chemistry [12], a computational model of particle swarms following certain kinetic rules is a good example for a swarm pattern formation approach that can self-organise and self-repair. However, it requires velocity of nearby particles, which is difficult to obtain, and it is not straightforward to design the kinetic rules producing the desired patterns. Mamei et al. [7] used the concept of morphogen gradient diffusion to achieve pattern formation, but patterns are limited to polygonal shapes, and robots are assumed to be able to pass through each other without a physical size. A hierarchical two-layer GRN (H-GRN) is introduced for target entrapping, where the first layer is responsible for adaptive pattern generation, while the second is a control mechanism that drives the robots on to the generated pattern [13]. This H-GRN concept is demonstrated by extracting a certain number of points from an entrapping pattern and guiding robots to a nearest point. This one-to-one matching between robots and points could be a hard constraint in uncertain and dynamic environments. Cheah et al. [5] have introduced a region-based shape control based on potential field, where each robot in the group stays within a region as a group while maintaining a minimum distance from each other. It addressed the convergence analysis, and considered various shapes of the desired region. However, the target shape needs to be predefined with limited complexity, which may become inadequate for handling unknown environmental changes.

To address the above limitations of the existing work, this paper proposes a morphogenetic approach to a target entrapping problem by integrating a two layer H-GRN with the region-based shape control for swarm robots. The morphogenetic approach to collective systems exploits the genetic and cellular mechanisms that govern biological morphogenesis [10] which refers to the biological process in which cells divide, grow and differentiate, and finally resulting in the mature morphology under GRNs and morphogens. Here, GRNs are models of genes and the interaction of gene products that describe the gene expression dynamics [14]. Morphogen gradients, concentration gradients of substances present in the environment, play an important role to trigger the specific gene expression in morphogenesis. The upper layer of the proposed H-GRN is responsible for pattern generation which provides a morphogen gradient to the lower layer. The lower layer implements a region-based shape control strategy that guides all robots into the desired region from the upper layer, and maintains a specified minimum distance between each robot and its neighbouring robots as well. The minimum distance between robots is adapted to evenly distribute robots into the desired region. Using this region-based shape control for target entrapment instead of boundary coverage as in our previous work enables robots to entrap the targets more tightly and herd them into a desired shape or position by directly contacting and pushing the targets.

The rest of this paper is structured as follows. Section 2 presents a problem statement including the connection between multicellular organisms and multi- 
agent systems. Section 3 introduces a H-GRN model with a region-based shape control strategy, consisting of an upper layer for region generation and a lower layer for robot guidance into the region. Section 4 presents numerical simulation results from scenarios containing either pre-defined stationary or moving targets. Conclusions and future work are given in Section 5.

\section{Problem Statement}

This paper considers the problem of entrapping stationary or moving targets using a swarm of robots, consisting of two tasks: region generation and region-based shape control. Based on the target location, the entrapping region is generated, and the robots are deployed into the generated region. Similar to [13], we distinguish between organising robots that can detect at least one target and are responsible for the region generation, and non-organising robots that have not yet detected any target. The non-organising robots will follow their neighbouring robots until they detect a target or receive information on the target from organising robots. It is assumed that global (or at least local) 2D position is available for the robots, and when the robots are close to the targets, they can obtain the position of targets either by direct sensing or from other organising robots through local communication.

As we exploit the GRNs found in multicellular organisms to control swarm robots, a metaphor between cells and robots is required. To this end, each cell is considered as a single robot where protein concentrations of genes in the cell correspond to the position and internal states. Each protein has the following three roles: i) auto-regulation which regulate the gene expression level that produces the protein, thus controlling the robot's behaviour ii) reaction to a certain morphogen gradient from the environment (in this paper, from targets to be entrapped), and iii) diffusion into other cells to avoid collision of the robots. In the following sections, we will show in detail how to use these GRN functions for dealing with a target entrapping problem.

\section{H-GRN Model with Region-Based Shape Control}

This study uses a two-layer hierarchical GRN (H-GRN) model [13], however, with a different objective of region-based shape formation for reliable target entrapping, as illustrated in Fig. 1. The upper layer of the H-GRN is for pattern generation that provides a desired entrapping pattern based on local sensory inputs that report the position of the targets. In the figure, the protein concentration $p$ represents the environmental input (i.e. target positions), which will serve as the input of the upper GRN and activate $g_{1}, g_{2}$ and $g_{3}$. In particular, the concentration of $g_{3}$ takes the role of morphogen to form the desired region or shapes around targets which will be transmitted to the lower layer. Note that the dynamics of the GRN in the upper layer is activated only in the organising robots that are able to detect targets, while the non-organising robots simply follow the movement of neighbouring organising robots. Once the target pattern 
Environment input

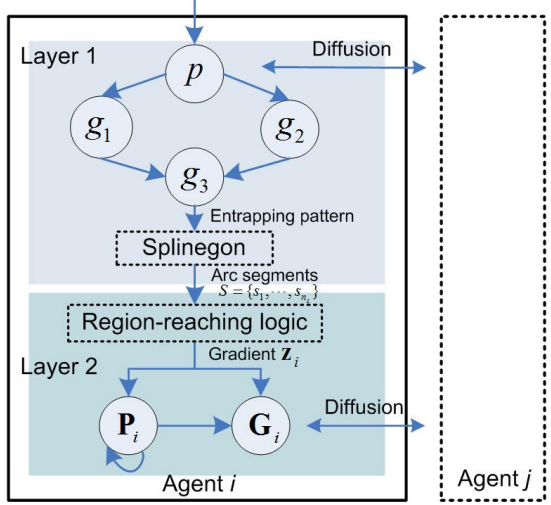

Fig. 1. Illustration of a two-layer H-GRN structure for target entrapping

is generated by the upper layer, it will function as the input to the lower layer to trigger its dynamics. The lower layer is for region-based shape control to guide all robots into the desired region while maintaining a specified minimum distance between the robots. These functions are realised by a single GRN where proteins $\mathbf{G}$ and $\mathbf{P}$ are used to represent the current positions and internal states of the robots. Thanks to diffusion of proteins $p$ and $\mathbf{G}$, the neighbouring robots can share the target information and maintain a desired distance between robots to avoid robot collision.

\subsection{Upper layer: Region generation}

Target entrapping pattern For generation of the target entrapping pattern, each organising robot will utilise the following gene regulatory dynamics to generate the desired concentrations:

$$
\begin{aligned}
\frac{d p_{j}}{d t} & =-p_{j}+\nabla^{2} p_{j}+\gamma_{j}, \quad p=\sum_{j=1}^{n_{t}} p_{j}, \\
\frac{d g_{1}}{d t} & =-g_{1}+\operatorname{sig}\left(p, \theta_{1}, k\right), \\
\frac{d g_{2}}{d t} & =-g_{2}+\left[1-\operatorname{sig}\left(p, \theta_{2}, k\right)\right], \\
\frac{d g_{3}}{d t} & =-g_{3}+\operatorname{sig}\left(g_{1}+g_{2}, \theta_{3}, k\right), \\
\operatorname{sig}(x, z, k) & =\frac{1}{1+e^{-k(x-z)}},
\end{aligned}
$$

where $p_{j}$ represents the protein concentration produced by the $j$-th target input $\gamma_{j}$, and $p$ the sum of concentrations of all detected $n_{t}$ targets. The integrated protein $p$ will activate the internal protein concentrations. $k$ is a positive constant 


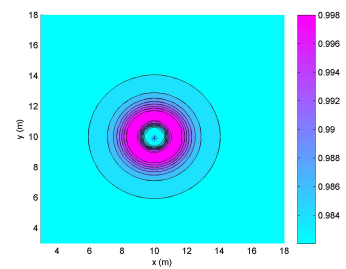

(a) $\theta_{3}=0.8$

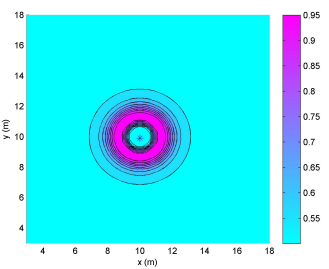

(b) $\theta_{3}=1.0$

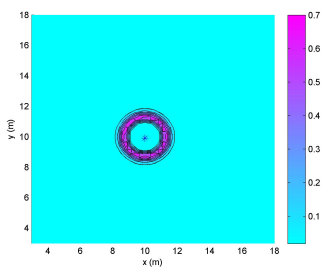

(c) $\theta_{3}=1.2$

Fig. 2. Examples of a target entrapping pattern from protein concentration $g_{3}$ generated by the upper layer of the H-GRN according to different threshholds $\theta_{3}$ with fixed $\theta_{1}=0.25$ and $\theta_{2}=0.3$ where target is located at $(x, y)=(10,10)$.

which determines the slope of sigmoid function, and $g_{1}, g_{2}$ and $g_{3}$ are protein concentrations, where $g_{3}$ defines target entrapping pattern. Note that $g_{3}$ is regulated by both $g_{1}$ and $g_{2}$ that are regulated by $p$ only within a particular range of its concentration. In particular, protein $g_{3}$ can be regulated by protein $p$ only when the concentration of $p$ is between $\theta_{1}$ and $\theta_{2}$. This leads to an activating band of circle shapes depending on the thresholds of a sigmoid function $\theta_{1}, \theta_{2}$ and $\theta_{3}$, as shown in Fig. 2 .

Splinegone representation For the generated entrapping pattern to be used by the lower layer of the H-GRN, the contour of the target pattern needs to be extracted. This work uses a subset of a class of object termed Splinegons [15] to create a set of vertices that are connected by line segments of constant curvature, representing the curved nature of the target pattern as a set of arc segments. Firstly, a few representative points whose $g_{3}$ concentration is higher than a threshold value on the pattern are selected to describe the target pattern, and those points are connected by line segments of constant curvature. This allows us to form shape constraints to be conveniently used for a region-based shape control. The mathematical details for the constructing the whole Splinegon can be found in [15]. Figure 3 shows the procedure for Splinegon representation from a given target pattern using selected representative points and constant curvature generation. The generated region from the upper layer of the H-GRN can then be modelled as a set of circular arc segments $s_{l} \in S=\left\{s_{1}, \cdots, s_{n_{s}}\right\}$. For each arc segment, only the centre position and its curvature will be used for region-based shape control in the next section.

\subsection{Lower layer: region-based shape control}

The lower layer of the H-GRN aims to guide all robots into the desired region generated by the upper layer and to maintain a specified minimum distance between robots. In the following, we first describe the GRN dynamics for driving the robots to the target region and then present the region-based shape control logic. At the end of this section, we discuss issues for implementing the regionbased shape control within the GRN framework. 


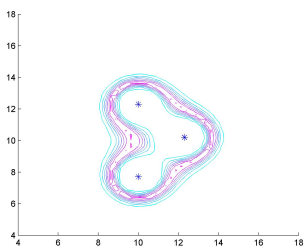

(a) Gradient value from $g_{3}$

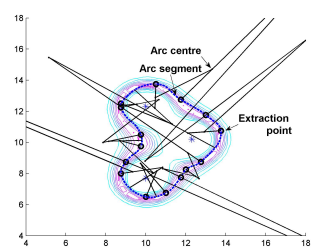

(b) Curvature segments

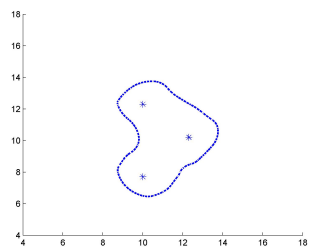

(c) Splinegon

Fig. 3. Splinegon representation procedure from a target entrapping pattern generated by upper layer where targets are represented as a blue star.

GRN dynamics The GRN in the lower layer is the same as the one used in our previous work [13], which was a modified GRN model used in [16]:

$$
\begin{aligned}
\frac{d \mathbf{G}_{i}}{d t} & =-a \mathbf{z}_{i}+m \mathbf{P}_{i} \\
\frac{d \mathbf{P}_{i}}{d t} & =-c \mathbf{P}_{i}+r f\left(\mathbf{z}_{i}\right)+b \mathbf{D}_{i}
\end{aligned}
$$

where $i \in\left\{1, \cdots, N_{o}\right\}$ is the index of an organising robot, and protein types $\mathbf{G}_{i}$ and $\mathbf{P}_{i}$ correspond to a $2 \mathrm{D}$ position and internal state vector of robot $i$, respectively. $a, m, c, r$ and $b$ are constants to be optimised depending on the objectives of the task. $\mathbf{D}_{i}$ represents the concentration of protein $\mathbf{G}$ diffused out of the cell, indicating the density of robots and obstacles in the neighbourhood:

$$
\mathbf{D}_{i}=\sum_{j=1}^{n_{i}} \mathbf{D}_{i}^{j}
$$

where $n_{i}$ denotes the number of robots in the neighbourhood of robot $i$, and $\mathbf{D}_{i}^{j}$ represents the diffused protein concentration vector from robot $j$ :

$$
\mathbf{D}_{i}^{j}=\frac{\mathbf{G}_{i}-\mathbf{G}_{j}}{\left\|\mathbf{G}_{i}-\mathbf{G}_{j}\right\|} .
$$

The diffusion process is activated only when the distance to the neighbour is less than a threshold $r_{n}$. In order to embed the desired shape into the regulatory dynamics, $f\left(\mathbf{z}_{i}\right)$ is defined as the following sigmoid function:

$$
f\left(\mathbf{z}_{i}\right)=\frac{1-e^{\alpha \mathbf{z}_{i}}}{1+e^{\alpha \mathbf{z}_{i}}}
$$

where $\mathbf{z}_{i}$ represents a gradient value at the robot's current position, and $\alpha>0$ determines the slope of the sigmoid function. This $\mathbf{z}_{i}$ regulates the concentration of both proteins $\mathbf{G}$ and $\mathbf{P}$ as a feedforward input in the GRN dynamics as in Eq. (7) so that robots could form a desired shape, which is defined as:

$$
\mathbf{z}_{i}=\frac{d h_{i}}{d \mathbf{G}_{i}}
$$


where $h_{i}$ is the desired shape on which the robots need to be deployed defined by the upper layer. For instance, if the robots need to be deployed onto a circle of a radius $R$ at a point $\mathbf{c}=\left[c_{x}, c_{y}\right]^{T}$, the shape function can be defined as:

$$
h_{i}=\left[\left(G_{i, x}-c_{x}\right)^{2}+\left(G_{i, y}-c_{y}\right)^{2}-R^{2}\right]^{2} .
$$

Note that if a circle radius $R$ approaches to zero, then the robot is to be deployed onto a point $\mathbf{c}$ rather than a circle.

Region-Reaching control logic The region-based shape control logic is developed in which the desired region is specified by an inequality function as follows:

$$
F(X) \leq 0,
$$

where $F(X)$ is a continuous scalar function with continuous first partial derivatives. In this paper, the target pattern is represented as a set of arc segments:

$$
S_{l}\left(\mathbf{G}_{i}\right)=\left(G_{i, x}-c_{l, x}\right)^{2}+\left(G_{i, y}-c_{l, y}\right)^{2}-\left(\frac{1}{\kappa_{l}}\right)^{2}=0
$$

where $\left[c_{l, x}, c_{l, y}\right]^{T}$ and $\kappa_{l}$ are the centre position and the curvature of the $l$-th arc segment, respectively. Thus, inequality functions for region shape control can be defined by $S_{l}\left(\mathbf{G}_{i}\right) \leq 0$ for keeping robots inside an arc segment or $-S_{l}\left(\mathbf{G}_{i}\right) \leq 0$ for pushing robots towards outside an arc. Inequality functions for the entire complex shape using the Splinegon representation can then be expressed as:

$$
F\left(\mathbf{G}_{i}\right)=\left[ \pm S_{1}\left(\mathbf{G}_{i}\right), \pm S_{2}\left(\mathbf{G}_{i}\right), \cdots, \pm S_{n_{s}}\left(\mathbf{G}_{i}\right)\right]^{T} \leq 0 .
$$

In this study, for an efficient region shape control, each robot finds its nearest arc segment $s_{l_{i}^{*}}$ at its current location rather than considering all arc segments consisting of the Splinegon:

$$
s_{l_{i}^{*}}=\arg \min _{s_{l} \in S}\left(\left\|\mathbf{G}_{i}-s_{l}^{m}\right\|\right),
$$

where $s_{l}^{m}$ is the middle point of $l$-th arc segment. Depending on the sign of the arc curvature and the position of targets, the shape control logic is divided into three cases with a gradient $\mathbf{z}_{i}$ and the inequality function of the nearest arc segment as follows.

Case 1: $\kappa_{l_{i}^{*}} \leq 0$

$$
\mathbf{z}_{i}= \begin{cases}0, & \text { if } S_{l_{i}^{*}}\left(\mathbf{G}_{i}\right) \leq 0 \\ \frac{d h_{1, i}}{d \mathbf{G}_{i}}, & \text { otherwise }\end{cases}
$$

where $h_{1, i}=\left[\left(G_{i, x}-c_{l_{i}^{*}, x}\right)^{2}+\left(G_{i, y}-c_{l_{i}^{*}, y}\right)^{2}\right]^{2}$. This gradient drives a robot towards the centre of its nearest arc segment, and once the robot enters a circular sector of a desired Splinegon, the gradient becomes zero, as illustrated in Fig. 4. 


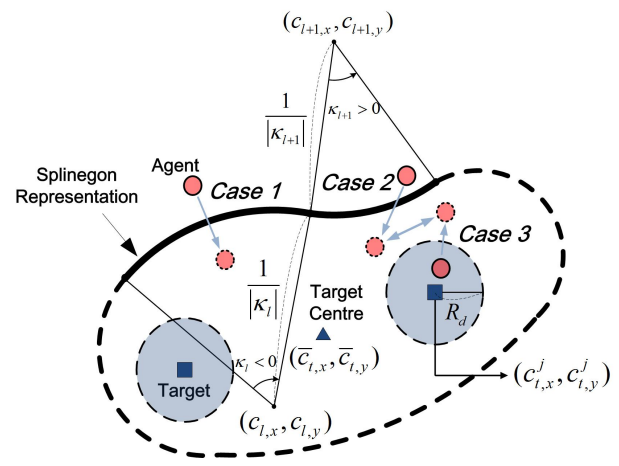

Fig. 4. Geometric relations for the region-based shape control logic.

Case 2: $\kappa_{l_{i}^{*}}>0$

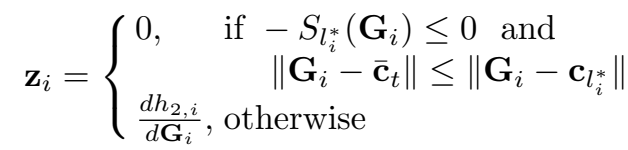

where $h_{2, i}=\left[\left(G_{i, x}-\bar{c}_{t, x}\right)^{2}+\left(G_{i, y}-\bar{c}_{t, y}\right)^{2}\right]^{2}$. Here, $\overline{\mathbf{c}}_{t}=\left[\bar{c}_{t, x}, \bar{c}_{t, y}\right]^{T}$ is the centre position of a target group. This gradient brings a robot towards the centre of a target group instead of an arc segment which is outside the Splinegon. Only if a robot is outside a circular sector $\left(-S_{l_{i}^{*}}\left(\mathbf{G}_{i}\right) \leq 0\right)$ as well as closer to the centre of target group than the one of an arc (i.e. $\left\|\mathbf{G}_{i}-\overline{\mathbf{c}}_{t}\right\| \leq\left\|\mathbf{G}_{i}-\mathbf{c}_{l_{i}^{*}}\right\|$ ), the gradient is zero.

Case 3: $\left\|\mathbf{G}_{i}-\mathbf{G}_{t}^{j}\right\|<R_{d}$ (for all $j \in\left\{1, \cdots, n_{t}\right\}$, regardless of the sign of $\left.\kappa_{l_{i}^{*}}\right)$

$$
\mathbf{z}_{i}=\frac{d h_{3, i}}{d \mathbf{G}_{i}}
$$

where $h_{3, i}=\left[(1+\epsilon) R_{d}^{2}-\left(G_{i, x}-c_{t, x}^{j}\right)^{2}-\left(G_{i, y}-c_{t, y}^{j}\right)^{2}\right]^{2}$. Here, $\epsilon$ is a positive constant. If a robot is inside a certain circular boundary of $R_{d}$ around the target, the robot is pushed out of that area (which is larger than $R_{d},(1+\epsilon) R_{d}$ ), in order to avoid any collision with the targets.

\subsection{Implementation issues}

In order to implement the proposed algorithm in real robots in a changing environment, there are several issues to be addressed. First, the robots should be able to localise themselves with their own onboard sensors such as encoders or an inertial navigation system. Note that this localisation can be done in a local coordinate system via robot-robot communications by choosing a reference point as the origin. Second, as the total number of robots and targets is unknown to each robot, an appropriate neighbourhood size $r_{n}$ for the diffusion process in Eq. (8) cannot be pre-defined in practice to ensure the convergence 
of the system or distribute the robots in the region as evenly as possible. This study adopts a similar method used in [17], which adjusts the initial guess of the distance with two physical constraints of the robot: the bumper range $d_{\min }$ and the sensor range $d_{\max }$. If the current neighbour distance $d$ is found to be small, then it is updated with a half-sum of $d$ and $d_{\max }$ resulting in a distance increase, whereas if the distance is large, it is updated with $d_{m i n}$. Whether the current distance is close to optimal or not is determined by calculating the average number of neighbours after a sufficiently large time elapse for robots to stabilise inside the region for several iterations. Lastly, the effect of communication/sensing noise and delay should be carefully considered as this might lead to performance degradation or even instability of the shape control.

\section{Numerical Simulations}

Numerical simulations have been performed using scenarios containing either stationary or moving targets to validate the feasibility and benefit of the proposed algorithm. The number of robots used in the simulation is 100. Parameters for the upper layer of the H-GRN are set up as $\theta_{1}=0.25, \theta_{2}=0.3, \theta_{3}=1.2$ and $k=20$, and for the lower layer as $a=6.5, m=4.2, c=9.9, r=4.3$ and $b=3.5$. These values are obtained by an evolutionary optimisation run explained in [13] and fine-tuned for stabilisation of robots inside the desired region within a reasonable time. In addition, the maximum speed of the robot is bounded by $0.8 \mathrm{~m} / \mathrm{s}$ considering the robots' physical capability.

\subsection{Forming a pre-defined target region}

To entrap a stationary target, a pre-defined simple ring shape is first employed, assuming that the target is at the centre of a circle. Radius is 0.2 and $0.5 \mathrm{~m}$ for inner and outer circles, respectively, to define a region. A desired distance between the robots is $0.065 \mathrm{~m}$, and the one between robots and obstacles is $0.3 \mathrm{~m}$. Figure 5 shows snapshots of a situation where 100 robots are randomly distributed in the space, form the target region driven by the H-GRN, and then avoid two moving obstacles. By using a diffusion term $\mathbf{D}$ between robots and obstacles as in Eqs. (7) (9), the GRN dynamics inherently adapts itself to the environmental changes, i.e., the moving obstacles. The movie clip for this can be downloaded at dl.dropboxusercontent. com/u/17047357/Ring_Obstacle.wmv.

In the following, we consider entrapment and tracking of a single moving target. If the trajectory of the target is known or can be estimated, it can be embedded in the GRN dynamics by modifying Eq. (6) as:

$$
\frac{d \mathbf{G}_{i}}{d t}=-a \mathbf{z}_{i}+m \mathbf{P}_{i}+\mathbf{v}_{t}
$$

where $\mathbf{v}_{t}$ is the velocity of the target to be followed. Figure 6 shows the numerical simulation result on tracking a moving target using a ring shape with or without information about the target movement. The trajectory of the moving target is 


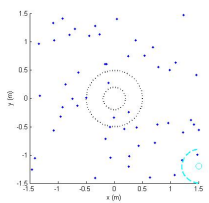

(a) $\mathrm{T}=0 \mathrm{~s}$

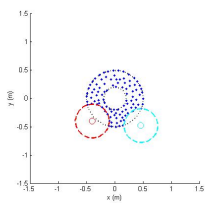

(b) $\mathrm{T}=8 \mathrm{~s}$

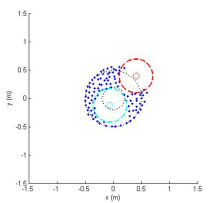

(c) $\mathrm{T}=12 \mathrm{~s}$



(d) $\mathrm{T}=16 \mathrm{~s}$

Fig. 5. 100 robots (blue points) forming a target shape denoted as a ring and avoiding moving obstacles, which are denoted by a red and a cyan small circle.

denoted by a red line, which is specified by $\mathbf{c}_{t}=\left[c_{x}, c_{y}\right]^{T}=0.1[t \quad 3 \sin (t / 3)]^{T}$, where $t$ represents time in seconds. It is assumed that each robot is equipped with a sensor that gives the range and direction to the target at $10 \mathrm{~Hz}$. Sensory noise is set to zero-mean Gaussian noise with a standard deviation of $\sigma_{r}=0.2$ $\mathrm{m}$ for the range and $\sigma_{\phi}=2^{\circ}$ for the direction. To obtain accurate estimates of the target motion, the decentralised extended information filter (DEIF) [18] is applied with a constant velocity target model. If the target velocity is unknown, it appears to be difficult for the swarm of robots to closely follow the moving target while forming the desired ring shape as shown in Fig. 6(a). By contrast, if the target position and velocity can be estimated by the DEIF filter, the robots successfully entraps the moving target, as shown in Fig. 6(b).

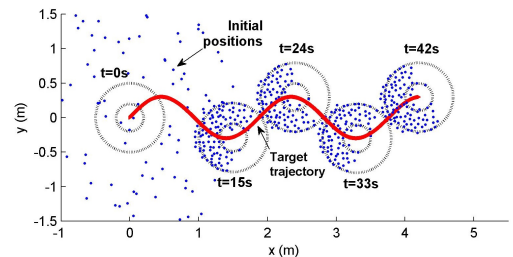

(a)

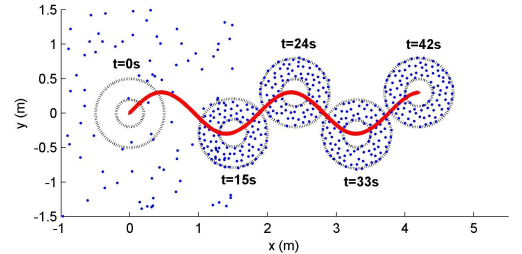

(b)

Fig. 6. Forming a ring-shape while tracking a target (a) The target position is known but the velocity is unknown. (b) The target position and the velocity are estimated.

\subsection{Adaptive formation of complex regions}

To entrap multiple targets, the desired region generated by the upper layer of the H-GRN may become very complex and needs to be represented using Splinegon. Figure 7 (a) shows a case of multiple stationary targets with an initial guess of a neighbourhood size of $0.55 \mathrm{~m}$, which fails to stabilise the motion inside the region and maintain the given distance between robots. By adjusting the neighbourhood size with $d_{\max }=1.0 \mathrm{~m}$ and $d_{\min }=0.1 \mathrm{~m}$ through iterations of adaptation as shown in Fig. 7(c), the final neighbourhood size of $0.4234 \mathrm{~m}$ is obtained resulting in an even distribution of the robots, as shown in Fig. 7(b).

Finally, Figure 8 shows the entrapping of multiple moving targets with a changing complex shape. As the targets move away from each other, the desired 


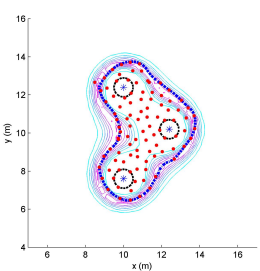

(a) Iteration $=1$



(b) Iteration $=6$

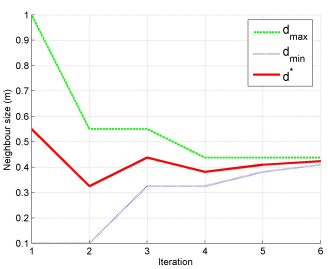

(c) Distance adaptation

Fig. 7. Entrapping of multiple static targets with neighbourhood size adaptation. By adjusting the neighbourhood size through iterations using the bumper range $d_{\min }$ and the maximum sensor range $d_{\max }$, the final size of $0.4234 \mathrm{~m}$ is obtained at iteration 6 .

shape is dynamically changing from the upper layer of the H-GRN. After 20 seconds, the targets stop moving, and the robots are able to organise themselves into a region that surrounds the targets, as shown in Fig. 8(c). The movie clip can be downloaded at dl.dropboxusercontent.com/u/17047357/Dynamic_Target.wmv.



(a) $\mathrm{T}=0 \mathrm{~s}$

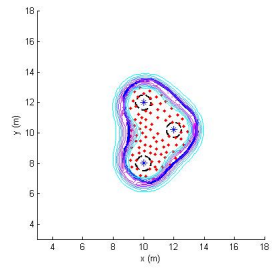

(b) $\mathrm{T}=15 \mathrm{~s}$

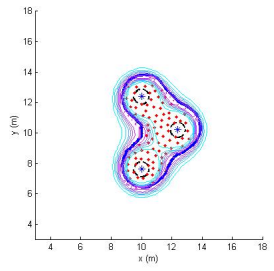

(c) $\mathrm{T}=35 \mathrm{~s}$

Fig. 8. Entrapping of multiple moving targets with a changing complex region. As the targets move away from each other, an entrapping shape is changing accordingly, and consequently the robots are organising themselves inside a shape.

\section{Conclusions and Future Work}

This paper presented a morphogenetic approach to region formation for entrapping targets using a swarm of robots. By adopting the H-GRN structure, the proposed algorithm has shown to be adaptable to environmental changes resulting from unknown target movements or obstacles. This capability of entrapping stationary or moving targets by forming a region can be applied to a variety of tasks such as contaminant/hazardous material boundary monitoring or isolation and transporting/herding targets into a pre-defined pattern or goal position. As future work, the idea of evolving the H-GRN based on simple network motifs for more flexible and robust pattern generation will be investigated, instead of using a predefined structure as in this paper. Moreover, relaxation of the assumption in the present model that relies on organising robots will be studied for a more realistic distributed self-organising system. 
Acknowledgments. This work was funded by the European Commission 7th Framework Program, Project No. 601062, SWARM-ORGAN.

\section{References}

1. J.R. Lawton, R.W. Beard, and B.J. Young. A decentralized approach to formation maneuvers. IEEE Transactions on Robotics and Automation, 19(6):933-941, 2003.

2. T. Balch and R.C. Arkin. Behavior-based formation control for multirobot teams. IEEE Transactions on Robotics and Automation, 14(6):926-939, 1998.

3. R. Alur, A. Das, J. Esposito, R. Fierro, Y. Hur, G. Grudic, V. Kumar, I. Lee, J. P. Ostrowski, G. Pappas, J. Southall, J. Spletzer, and C. J. Taylor. A framework and architecture for multirobot coordination. Experimental Robotics VII, Lecture Notes in Control and Information Sciences, 271:303-312, 2001.

4. L. Consolini, F. Morbidi, F. Prattichizzo, and M. Tosques. Leader-follower formation control of nonholonomic mobile robots with input constraints. Automatica, 44(5):1343-1349, 2008.

5. M. Hsieh, V. Kumar, and L. Chaimowicz. Decentralized controllers for shape generation with robotic swarms. Robotica, 26(5):691-701, 2008.

6. R. Olfati-Saber. Flocking for multi-agent dynamic systems: Algorithms and theory. IEEE Transactions on Automatic Control, 51(3):401-420, 2006.

7. M. Mamei, M. Vasirani, and F. Zambonelli. Experiments of morphogenesis in swarms of simple mobile robots. Applied Artificial Intelligence, 18:903-919, 2004.

8. K. Yeom and J-H. Park. Artificial morphogenesis for arbitrary shape generation of swarms of multi agents. In IEEE Fifth International Conference on Bio-Inspired Computing: Theories and Applications (BIC-TA), pages 509 - 513, 2010.

9. W.-M. Shen, P. Will, and A. Galstyan. Hormone-inspired self-organization and distributed control of robotic swarms. Autonomous Robots, 17:93-105, 2004.

10. Y. Jin and Y. Meng. Morphogenetic robotics: An emerging new field in developmental robotics. IEEE Transactions on Systems, Man, and Cybernetics, Part C: Applications and Reviews, 41(2):145-160, 2011.

11. T. Taylor, P. Ottery, and J. Hallam. Pattern formation for multi-robot applications: Robust, self-repairing systems inspired by genetic regulatory networks and cellular self-organisation. Informatics Research Report, 2006.

12. H. Sayama. Robust morphogenesis of robotic swarms. IEEE Computational Intelligence Magazine, 5(3):43-49, 2010.

13. Y. Jin, H. Guo, and Y. Meng. A hierarchical gene regulatory network for adaptive multirobot pattern formation. IEEE Trans. on Systems, Man, And CyberneticsPart B: Cybernetics, 42(3):805-816, 2012.

14. H. De Jong. Modeling and simulation of genetic regulatory systems: A literature review. Journal of Computational Biology, 9(1):67-103, 2002.

15. B.A. White, A. Tsourdos, I. Ashokaraj, S. Subchan, and R. Zbkowski. Contaminant cloud boundary monitoring using network of UAV sensors. IEEE Sensors Journal, 8(10):1681-1692, 2008.

16. I. Salazar-Ciudad, J. Jernvall, and S. Newman. Mechanisms of pattern formation in development and evolution. Development, 130(10):2027-2037, 2003.

17. H. Guo, Y. Jin, and Y. Meng. A morphogenetic framework for self-organized multirobot pattern formation and boundary coverage. ACM Trans. on Autonomous and Adaptive Systems, 7(1):15:1-23, 2012.

18. A.G.O. Mutambara. Decentralized Estimation and Control for Multisensor Systems. CRC Press LLC, Boca Raton, Florida, 1998. 